\title{
Synthesis of (2-chloroquinolin-3-yl)-1,3,4-thiadiazole-2-carboxamides*
}

\author{
A. N. Aksenov, M. M. Krayushkin, and V. N. Yarovenko ${ }^{\star}$ \\ N. D. Zelinsky Institute of Organic Chemistry, Russian Academy of Sciences, \\ 47 Leninsky prosp., 119991 Moscow, Russian Federation. \\ E-mail: yarov@ioc.ac.ru
}

\begin{abstract}
(2-Chloroquinolin-3-yl)-1,3,4-thiadiazole-2-carboxamides were synthesized from hydrazones obtained via the reaction of 3-formyl-2-chloroquinoline with oxamic acid thiohydrazides.
\end{abstract}

Key words: heterocyclic compounds, quinolines, hydrazones, 1,3,4-thiadiazoles, oxamic acid thiohydrazides.

Quinolines are applied in the synthesis of various drugs (Ofloxacin, Norfloxacin, Ciprofloxacin, Chloroquine, etc.) and some compounds possessing a wide range of biological activity. ${ }^{1,2}$ A quinoline derivative (hydroxychloroquine) has been proposed for the treatment of COVID-19. ${ }^{3}$ Considerable attention is paid to the synthesis and biological activity of 2-chloroquinolines, starting from which substances exhibiting antimicrobial, antimalarial, antiinflammatory, antitumor, and antiparasitic activities have been obtained. ${ }^{\mathbf{4} 5}$ It is known ${ }^{\mathbf{6 , 7}}$ that $1,3,4$-thiadiazole derivatives are employed as promising pharmacophore moieties in the design of antibacterial, antifungal, antituberculosis, and antitumor drugs. Taking into account the wide range of biological activity of these heterocycles, it seems reasonable to design compounds containing both quinoline and thiadiazole moieties in one molecule. ${ }^{\mathbf{8}-10}$ In the present work, we proposed a method for the synthesis of new (i.e., never reported) (2-chloroquinolin-3yl)-1,3,4-thiadiazoles bearing carboxamide groups.

We have earlier demonstrated ${ }^{\mathbf{1 1}}$ a high synthetic potential of oxamic acid thiohydrazides in the synthesis of various heterocyclic compounds. According to our ${ }^{\mathbf{1 2}}$ approach (Scheme 1) that involves the reaction of hydrazine hydrate with 2-morpholinothioxoacetamides $\mathbf{3 a - d}$, which were obtained from $\alpha$-chloroacetamides $\mathbf{2 a - d}$ and a preliminarily prepared solution of elemental sulfur in morpholine, oxamic acid thiohydrazides $\mathbf{4 a - d}$ were synthesized.

It has been demonstrated $\mathbf{~}^{\mathbf{3}}$ that hydrazones of oxamic acid thiohydrazides can exist in a solution as an equilibrium mixture of the two forms, linear $\mathbf{A}$ and cyclic $\mathbf{B}$, due to the ring-chain tautomerism (Scheme 2). The ratio of isomers depends on the nature of moieties in the thiohydrazide group in the hydrazones.

The reaction of 3-formyl-2-chloroquinoline 5 with oxamic acid thiohydrazides $\mathbf{4 a}-\mathbf{d}$ in ethanol leads to

* Dedicated to Academician of the Russian Academy of Sciences V. N. Charushin on the occasion of his 70th birthday.
Scheme 1

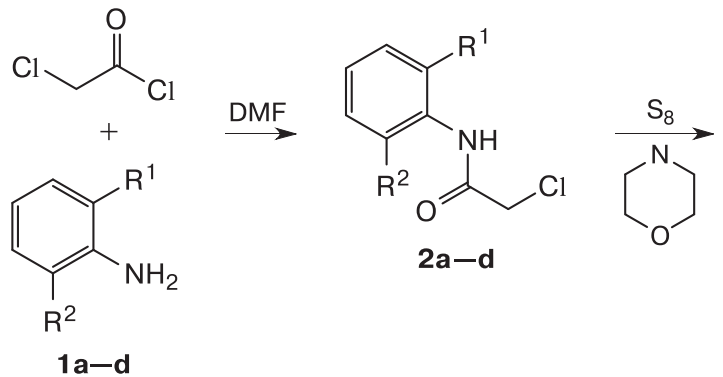<smiles>[R]c1cccc([R])c1NC(=O)C(=S)N1CCOCC1N</smiles>

$3 a-d$<smiles>[R]c1cccc([R])c1NC(=O)C(=S)NN</smiles>

$4 a-d$

1-4: $R^{1}=R^{2}=H(a) ; R^{1}=H, R^{2}=C l(b) ; R^{1}=H, R^{2}=M e(c)$; $R^{1}=R^{2}=M e(d)$

Scheme 2

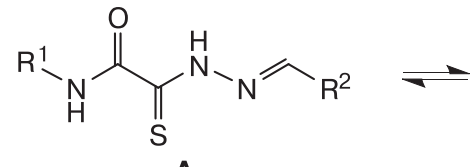

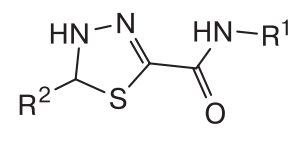

B

Published in Russian in Izvestiya Akademii Nauk. Seriya Khimicheskaya, No. 6, pp. 1131-1134, June, 2021. 
dihydrothiadiazoles $\mathbf{6 a - d}$ (Scheme 3). The interaction proceeds regioselectively, whereas the chlorine atom does not react with thiohydrazides.

\section{Scheme 3}

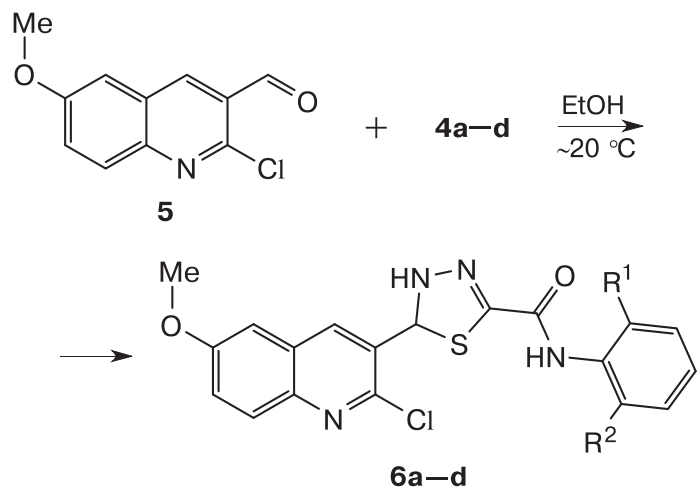

6: $R^{1}=R^{2}=H(a) ; R^{1}=H, R^{2}=C l(b) ; R^{1}=H, R^{2}=M e(c)$; $R^{1}=R^{2}=M e(d)$

Analysis of spectral data revealed that compounds 6a-d exist in a DMSO solution in the form of a single cyclic tautomer, viz., 4,5-dihydrothiadiazole-2-carboxamide. Instead of a hydrazinyl proton in the ${ }^{1} \mathrm{H}$ NMR spectrum, there is a singlet of proton at the $\mathrm{C}(5)$ atom of dihydrothiadiazole, observed in the range of $\delta 6.88-6.95$. The ${ }^{13} \mathrm{C}$ NMR spectrum contains a signal from the thiadiazolic $C(5)$ atom in the range of $\delta 71.10-71.69$, while there is no any signal of $\mathrm{C}=\mathrm{S}$ groups in the range of $\delta 180-200$.

The reaction of dihydrothiadiazoles $\mathbf{6 a}-\mathbf{d}$ with either acetic anhydride or acetyl chloride gave corresponding acylated dihydrothiadiazoles $7 \mathbf{a}-\mathbf{d}$ (Scheme 4).

\section{Scheme 4}

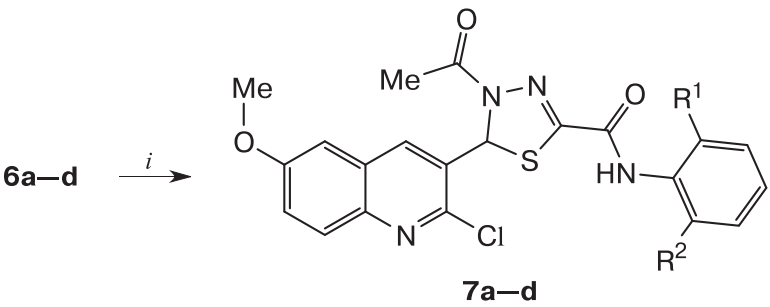

i. $\mathrm{Ac}_{2} \mathrm{O}$ or $\mathrm{AcCl}$, DMF.

7: $\mathrm{R}^{1}=\mathrm{R}^{2}=\mathrm{H}(\mathbf{a}) ; \mathrm{R}^{1}=\mathrm{H}, \mathrm{R}^{2}=\mathrm{Cl}(\mathbf{b}) ; \mathrm{R}^{1}=\mathrm{H}, \mathrm{R}^{2}=\mathrm{Me}(\mathbf{c})$; $R^{1}=R^{2}=M e(d)$

A singlet of methyl protons of the acetyl group appears in the ${ }^{1} \mathrm{H}$ NMR spectra of compounds $7 \mathbf{a}-\mathbf{d}$.

It should be noted that in contrast to our previous work ${ }^{\mathbf{1 4}}$ on the reactions of aromatic aldehydes with oxamic acid thiohydrazides, the autoxidation of dihydrothiadi- azoles $\mathbf{6 a - d}$ into the corresponding thiadiazoles does not occur. It was possible to convert compounds $\mathbf{6 a - d}$ into (2-chloroquinolin-3-yl)-1,3,4-thiadiazole-2-carboxamides $\mathbf{8 a - d}$ upon their treatment with DDQ in ethanol (Scheme 5).

\section{Scheme 5}<smiles>[R]c1cccc([R])c1NC(=O)c1nnc(-c2cc3cc(OC)ccc3nc2Cl)s1</smiles>

8: $R^{1}=R^{2}=H(a) ; R^{1}=H, R^{2}=C l(b) ; R^{1}=H, R^{2}=M e(c)$; $R^{1}=R^{2}=M e(d)$

In summary, we have proposed and verified a method for the preparation of previously unknown (2-chloroquinolin-3-yl)-1,3,4-thiadiazole-2-carboxamides from oxamic acid thiohydrazides.

\section{Experimental}

NMR spectra of compounds $\mathbf{6}-\mathbf{8}$ were recorded on a Bruker AM-300 instrument (at $300\left({ }^{1} \mathrm{H}\right)$ and $\left.75\left({ }^{13} \mathrm{C}\right) \mathrm{MHz}\right)$ in DMSO-d 6 . Mass spectra were recorded on a Varian MAT CH-6 instrument with a direct sample injection into the radiation source, the ionization energy was $70 \mathrm{eV}$, and the control voltage was $1.75 \mathrm{kV}$. High resolution mass spectra (HRMS) were recorded on a Bruker micrOTOF II instrument using electrospray as the ionization method. Melting points were measured using a Boetius apparatus without corrections. The reactions were monitored by TLC using Merck 60 F254 UV-254 chromatographic plates.

Synthesis of dihydrothiadiazoles $6 \mathbf{a}-\mathbf{d}$ (general procedure). A mixture of chloroquinolinecarbaldehyde $5(3.1 \mathrm{mmol})$ and thiohydrazide $\mathbf{4 a}-\mathbf{d}(3.5 \mathrm{mmol})$ in ethanol $(25 \mathrm{~mL})$ was stirred for $12 \mathrm{~h}$. The precipitate was filtered off and washed with a minimal amount of ethanol. This crude product was recrystallized from ethanol.

5-(2-Chloro-6-methoxyquinolin-3-yl)-4,5-dihydro- $N$-phenyl1,3,4-thiadiazole-2-carboxamide (6a). The yield was $63 \%$, tile red powder, m.p. $157-159^{\circ} \mathrm{C} .{ }^{1} \mathrm{H}$ NMR (DMSO-d 6 , $300.13 \mathrm{MHz}$ ), $\delta: 10.28(\mathrm{~s}, 1 \mathrm{H}, \mathrm{NH}$ in $\mathrm{PhCONH}) ; 9.51(\mathrm{~s}, 1 \mathrm{H}, \mathrm{NH}$ in the thiadiazole moiety); 8.33 (s, $1 \mathrm{H}, \mathrm{H}(4)$ in the quinoline moiety); $7.90(\mathrm{~d}, 1 \mathrm{H}, J=9.1 \mathrm{~Hz}) ; 7.73(\mathrm{~d}, 2 \mathrm{H}, J=7.9 \mathrm{~Hz}) ; 7.52(\mathrm{~d}, 1 \mathrm{H}$, $J=2.6 \mathrm{~Hz}) ; 7.47(\mathrm{dd}, 1 \mathrm{H}, J=9.3 \mathrm{~Hz}, J=2.8 \mathrm{~Hz}) ; 7.31(\mathrm{t}, 2 \mathrm{H}$, $J=7.9 \mathrm{~Hz}) ; 7.08(\mathrm{t}, 1 \mathrm{H}, J=7.4 \mathrm{~Hz}) ; 6.88(\mathrm{~s}, 1 \mathrm{H}, \mathrm{H}(5)$ in the thiadiazole moiety); 3.90 (s, $3 \mathrm{H}, \mathrm{MeO}) .{ }^{13} \mathrm{C} \mathrm{NMR}$ (DMSO-d 6 , $75 \mathrm{MHz}), \delta: 158.61,158.30,144.86,143.02,139.09,138.62$, $135.55,133.12,129.48,129.08,128.64,124.33,124.15,120.69$, $106.70,71.10(\mathrm{C}(5)$ in the thiadiazole moiety), $56.20(\mathrm{MeO})$. Found (\%): C, 57.39; H, 3.84; Cl, 8.72; N, 14.15; S, 8.11. $\mathrm{C}_{19} \mathrm{H}_{15} \mathrm{ClN}_{4} \mathrm{O}_{2}$ S. Calculated (\%): C, 57.21; H, 3.79; Cl, 8.89; $\mathrm{N}, 14.05 ; \mathrm{S}, 8.04$.

5-(2-Chloro-6-methoxyquinolin-3-yl)- $N$-(2-chlorophenyl)4,5-dihydro-1,3,4-thiadiazole-2-carboxamide (6b). The yield was $89 \%$, slightly pink powder, m.p. $199-201{ }^{\circ} \mathrm{C}$. ${ }^{1} \mathrm{H}$ NMR 
(DMSO- $\left.\mathrm{d}_{6}, 300.13 \mathrm{MHz}\right), \delta: 9.62(\mathrm{~s}, 1 \mathrm{H}, \mathrm{NH}$ in PhCONH); $9.58(\mathrm{~s}, 1 \mathrm{H}, \mathrm{NH}$ in the thiadiazole moiety); 8.35 (s, $1 \mathrm{H}, \mathrm{H}(4)$ in the quinoline moiety); $7.89(\mathrm{~d}, 1 \mathrm{H}, J=9.2 \mathrm{~Hz}) ; 7.87(\mathrm{~d}, 1 \mathrm{H}$, $J=7.0 \mathrm{~Hz}) ; 7.59-7.44(\mathrm{~m}, 3 \mathrm{H}) ; 7.36(\mathrm{t}, 1 \mathrm{H}, J=7.8 \mathrm{~Hz}) ; 7.22$ (t, $1 \mathrm{H}, J=7.7 \mathrm{~Hz}) ; 6.95(\mathrm{~s}, 1 \mathrm{H}, \mathrm{H}(5)$ in the thiadiazole moiety); $3.91(\mathrm{~s}, 3 \mathrm{H}, \mathrm{MeO}) .{ }^{13} \mathrm{C}$ NMR (DMSO-d 6 , $75 \mathrm{MHz}$ ), $\delta: 158.61$, 158.08, 144.81, 143.02, 138.00, 135.64, 134.42, 132.96, 129.93, 129.46, 128.64, 128.25, 126.91, 126.42, 125.01, 124.15, 106.78, $71.69(\mathrm{C}(5)$ in the thiadiazole moiety), 56.21 (MeO). Found (\%): $\mathrm{C}, 52.78 ; \mathrm{H}, 3.34 ; \mathrm{Cl}, 16.21 ; \mathrm{N}, 12.81 ; \mathrm{S}, 7.56 . \mathrm{C}_{19} \mathrm{H}_{14} \mathrm{Cl}_{2} \mathrm{~N}_{4} \mathrm{O}_{2} \mathrm{~S}$. Calculated (\%): C, 52.67; H, 3.26; Cl, 16.36; N, 12.93; S, 7.40.

5-(2-Chloro-6-methoxyquinolin-3-yl)-4,5-dihydro- $\mathrm{N}$-(2methylphenyl)-1,3,4-thiadiazole-2-carboxamide (6c). The yield was $91 \%$, beige powder, m.p. $213-214{ }^{\circ} \mathrm{C} .{ }^{1} \mathrm{H}$ NMR (DMSO-d 6 , $300.13 \mathrm{MHz}$ ), $\delta: 9.67$ (s, $1 \mathrm{H}, \mathrm{NH}$ in $\mathrm{PhCONH})$; 9.44 (s, $1 \mathrm{H}$, $\mathrm{NH}$ in the thiadiazole moiety); $8.37(\mathrm{~s}, 1 \mathrm{H}, \mathrm{H}(4)$ in the quinoline moiety); $7.90(\mathrm{~d}, 1 \mathrm{H}, J=9.1 \mathrm{~Hz}) ; 7.54(\mathrm{~d}, 1 \mathrm{H}, J=2.6 \mathrm{~Hz})$; $7.48(\mathrm{dd}, 1 \mathrm{H}, J=9.3 \mathrm{~Hz}, J=2.8 \mathrm{~Hz}) ; 7.39(\mathrm{~d}, 1 \mathrm{H}, J=7.6 \mathrm{~Hz})$; $7.28-7.09(\mathrm{~m}, 3 \mathrm{H}) ; 6.89$ (s, $1 \mathrm{H}, \mathrm{H}(5)$ in the thiadiazole moiety); 3.90 (s, $3 \mathrm{H}, \mathrm{MeO}) ; 2.21$ (s, $3 \mathrm{H}, \mathrm{CH}_{3}$ arom.). ${ }^{13} \mathrm{C} \mathrm{NMR}$ (DMSO-d 6 , $75 \mathrm{MHz}$ ), $\delta: 158.62,158.29,144.97,143.00,138.96$, $135.91,135.60,133.13,133.02,130.78,129.50,128.64,126.53$, $126.30,125.88,124.11,106.74,71.29(\mathrm{C}(5)$ in the thiadiazole moiety), 56.22 (MeO), $18.17\left(\mathrm{CH}_{3}\right.$ arom.). Found (\%): C, 58.29; $\mathrm{H}, 4.30 ; \mathrm{Cl}, 8.72 ; \mathrm{N}, 13.69 ; \mathrm{S}, 7.87 . \mathrm{C}_{20} \mathrm{H}_{17} \mathrm{ClN}_{4} \mathrm{O}_{2} \mathrm{~S}$. Calculated (\%): C, 58.18; H, 4.15; Cl, 8.59; N, 13.57; S, 7.77.

5-(2-Chloro-6-methoxyquinolin-3-yl)-4,5-dihydro- $\mathrm{N}$-(2,6dimethylphenyl)-1,3,4-thiadiazole-2-carboxamide (6d). The yield was $47 \%$, light yellow powder, m.p. $197-199{ }^{\circ} \mathrm{C}$. ${ }^{1} \mathrm{H}$ NMR (DMSO-d $6,300.13 \mathrm{MHz}$ ), $\delta: 9.72$ (s, $1 \mathrm{H}, \mathrm{NH}$ in PhCONH); 9.35 (s, $1 \mathrm{H}, \mathrm{NH}$ in the thiadiazole moiety); 8.43 (s, $1 \mathrm{H}, \mathrm{H}(4)$ in the quinoline moiety); $7.91(\mathrm{~d}, 1 \mathrm{H}, J=9.2 \mathrm{~Hz}) ; 7.56(\mathrm{~d}, 1 \mathrm{H}$, $J=2.6 \mathrm{~Hz}) ; 7.49(\mathrm{dd}, 1 \mathrm{H}, J=9.1 \mathrm{~Hz}, J=2.8 \mathrm{~Hz}) ; 7.08(\mathrm{~s}, 3 \mathrm{H})$; 6.89 (s, $1 \mathrm{H}, \mathrm{H}(5)$ in the thiadiazole moiety); 3.92 (s, $3 \mathrm{H}, \mathrm{MeO})$; $2.14\left(\mathrm{~s}, 6 \mathrm{H}, 2 \mathrm{CH}_{3}\right) .{ }^{13} \mathrm{C}$ NMR (DMSO-d 6 , $75 \mathrm{MHz}$ ), $\delta: 158.64$, $158.31,145.17,142.98,139.07,136.09,135.69,134.90,133.13$, $129.50,128.65,128.14,127.29,124.08,106.75,71.34(\mathrm{C}(5)$ in the thiadiazole moiety), 56.50, 56.23, $19.00\left(\mathrm{CH}_{3}\right), 18.52\left(\mathrm{CH}_{3}\right)$. Found (\%): C, 59.24; H, 4.65; Cl, 8.45; N, 13.37; S, 7.70. $\mathrm{C}_{21} \mathrm{H}_{19} \mathrm{ClN}_{4} \mathrm{O}_{2}$ S. Calculated (\%): C, 59.08; H, 4.49; Cl, 8.30; N, 13.12; S, 7.51.

Synthesis of acylthiadiazoles $7 \mathrm{a}-\mathrm{d}$ (general procedure $\boldsymbol{A}$ ). Thiohydrazide $\mathbf{4 a}-\mathbf{d}(0.75 \mathrm{mmol})$ was heated in acetic anhydride $(3 \mathrm{~mL})$ at $90{ }^{\circ} \mathrm{C}$ for $4 \mathrm{~h}$. The reaction mixture was treated with cold water $(20 \mathrm{~mL})$. Sodium carbonate was added until $\mathrm{pH} 6-7$, and the precipitate was filtered off and washed with water. The resulting products were recrystallized from ethanol or acetonitrile.

Synthesis of acylthiadiazoles $7 \mathbf{a}-\mathbf{d}$ (general procedure $B$ ). Acetyl chloride $(0.04 \mathrm{~g}, 0.45 \mathrm{mmol})$ was added to a solution of thiohydrazide $\mathbf{4 a}-\mathbf{d}(0.3 \mathrm{mmol})$ in DMF $(1 \mathrm{~mL})$. The mixture was stirred for $1 \mathrm{~h}$, water $(10 \mathrm{~mL})$ was added, and the formed precipitate was filtered off and washed on the filter with water, sodium carbonate solution ( $5 \%$ aq.), and water. The resulting products were recrystallized from ethanol or acetonitrile.

4-Acetyl-5-(2-chloro-6-methoxyquinolin-3-yl)-4,5-dihydro$\mathrm{N}$-phenyl-1,3,4-thiadiazole-2-carboxamide (7a). The yield was $85 \%(A), 70 \%(B)$, gray powder, m.p. $203-205{ }^{\circ} \mathrm{C}(\mathrm{EtOH})$. ${ }^{1} \mathrm{H}$ NMR (DMSO-d $\left.6,300.13 \mathrm{MHz}\right), \delta: 10.52(\mathrm{~s}, 1 \mathrm{H}, \mathrm{NH}$ in PhCONH); $8.20(\mathrm{~s}, 1 \mathrm{H}, \mathrm{H}(4)$ in the quinoline moiety); 7.90 $(\mathrm{d}, 1 \mathrm{H}, J=8.9 \mathrm{~Hz}) ; 7.70(\mathrm{~d}, 2 \mathrm{H}, J=8.0 \mathrm{~Hz}) ; 7.51(\mathrm{~s}, 1 \mathrm{H})$; $7.48(\mathrm{~d}, 1 \mathrm{H}, J=8.9 \mathrm{~Hz}) ; 7.51(\mathrm{~s}, 1 \mathrm{H}) ; 7.42(\mathrm{~s}, 1 \mathrm{H}) ; 7.38(\mathrm{t}, 1 \mathrm{H}$,
$J=7.3 \mathrm{~Hz}) ; 7.17$ (t, $1 \mathrm{H}, J=7.3 \mathrm{~Hz}) ; 3.89$ (s, $3 \mathrm{H}, \mathrm{MeO}) ; 2.58$ (s, $3 \mathrm{H}, \mathrm{MeCO}) .{ }^{13} \mathrm{C}$ NMR (DMSO-d 6 , $75 \mathrm{MHz}$ ), $\delta: 170.31$ $\left(\mathrm{C}=\mathrm{O}\right.$ in $\left.\mathrm{CH}_{3} \mathrm{CO}\right), 158.62,157.06,147.74,143.99,143.05$, $137.73,134.97,131.91,131.51,129.29,128.72,125.34,124.49$, $121.57,106.72,68.19(\mathrm{C}(5)$ in the thiadiazole moiety), 56.14 $\left(\mathrm{CH}_{3} \mathrm{O}\right), 22.68\left(\mathrm{CH}_{3}\right.$ in $\left.\mathrm{CH}_{3} \mathrm{CO}\right)$. Found (\%): C, 57.29; $\mathrm{H}, 3.91$; $\mathrm{Cl}, 8.22 ; \mathrm{N}, 12.84 ; \mathrm{S}, 7.38 . \mathrm{C}_{21} \mathrm{H}_{17} \mathrm{ClN}_{4} \mathrm{O}_{3} \mathrm{~S}$. Calculated (\%): C, 57.21; H, 3.89; Cl, 8.04; N, 12.71; S, 7.27.

4-Acetyl-5-(2-chloro-6-methoxyquinolin-3-yl)- $\mathrm{N}$-(2-chlorophenyl)-4,5-dihydro-1,3,4-thiadiazole-2-carboxamide (7b). The yield was $87 \%(A), 90 \%(B)$, light yellow powder, m.p. $252-253{ }^{\circ} \mathrm{C}$ (MeCN). ${ }^{1} \mathrm{H}$ NMR (DMSO-d $6,300.13 \mathrm{MHz}$ ), $\delta: 10.27$ (s, $1 \mathrm{H}$, $\mathrm{NH}$ in $\mathrm{PhCONH}) ; 8.25$ (s, $1 \mathrm{H}, \mathrm{H}(4)$ in the quinoline moiety); $7.90(\mathrm{~d}, 1 \mathrm{H}, J=9.2 \mathrm{~Hz}) ; 7.69(\mathrm{~d}, 1 \mathrm{H}, J=7.0 \mathrm{~Hz}) ; 7.62-7.29$ $(\mathrm{m}, 6 \mathrm{H}) ; 3.90\left(\mathrm{~s}, 3 \mathrm{H}, \mathrm{CH}_{3} \mathrm{O}\right) ; 2.55\left(\mathrm{~s}, 3 \mathrm{H}, \mathrm{CH}_{3} \mathrm{CO}\right) .{ }^{13} \mathrm{C} \mathrm{NMR}$ $\left(\mathrm{DMSO}_{\mathrm{d}}, 75 \mathrm{MHz}\right), \delta: 170.23\left(\mathrm{C}=\mathrm{O}\right.$ in $\left.\mathrm{CH}_{3} \mathrm{CO}\right), 158.62$, $157.17,146.90,143.95,143.04,135.03,133.88,131.33,130.18$, $129.47,129.00,128.73,128.49,128.32,127.60,124.50,106.77$, $68.64\left(\mathrm{C}(5)\right.$ in the thiadiazole moiety), $56.16\left(\mathrm{CH}_{3} \mathrm{O}\right), 22.61$ $\left(\mathrm{CH}_{3}\right.$ in $\left.\mathrm{CH}_{3} \mathrm{CO}\right)$. Found $(\%): \mathrm{C}, 53.16 ; \mathrm{H}, 3.44 ; \mathrm{Cl}, 14.79$; $\mathrm{N}, 11.89 ; \mathrm{S}, 6.81 . \mathrm{C}_{21} \mathrm{H}_{16} \mathrm{Cl}_{2} \mathrm{~N}_{4} \mathrm{O}_{3} \mathrm{~S}$. Calculated (\%): C, 53.06; $\mathrm{H}, 3.39 ; \mathrm{Cl}, 14.92 ; \mathrm{N}, 11.79 ; \mathrm{S}, 6.75$.

4-Acetyl-5-(2-chloro-6-methoxyquinolin-3-yl)-4,5-dihydro$\mathrm{N}$-(2-methylphenyl)-1,3,4-thiadiazole-2-carboxamide (7c). The yield was $88 \%(A), 79 \%(B)$, white powder, m.p. $193-194{ }^{\circ} \mathrm{C}$ (EtOH). ${ }^{1} \mathrm{H}$ NMR (DMSO-d $\left.6,300.13 \mathrm{MHz}\right), \delta: 10.22$ (s, $1 \mathrm{H}$, $\mathrm{NH}$ in $\mathrm{PhCONH}) ; 8.24$ (s, $1 \mathrm{H}, \mathrm{H}(4)$ in the quinoline moiety); $7.90(\mathrm{~d}, 1 \mathrm{H}, J=9.1 \mathrm{~Hz}) ; 7.56(\mathrm{~s}, 1 \mathrm{H}) ; 7.50(\mathrm{dd}, 1 \mathrm{H}, J=9.2 \mathrm{~Hz}$, $J=2.6 \mathrm{~Hz}) ; 7.42(\mathrm{~s}, 1 \mathrm{H}) ; 7.38-7.17(\mathrm{~m}, 4 \mathrm{H}) ; 3.91(\mathrm{~s}, 3 \mathrm{H}$, $\left.\mathrm{CH}_{3} \mathrm{O}\right) ; 2.56\left(\mathrm{~s}, 3 \mathrm{H}, \mathrm{CH}_{3} \mathrm{CO}\right) ; 2.24\left(\mathrm{~s}, 3 \mathrm{H}, \mathrm{CH}_{3}\right) .{ }^{13} \mathrm{C} \mathrm{NMR}$ $($ DMSO-d $6,75 \mathrm{MHz}), \delta: 170.28\left(\mathrm{C}=\mathrm{O}\right.$ in $\left.\mathrm{CH}_{3} \mathrm{CO}\right), 158.62$, 157.12, 147.40, 144.01, 143.02, 135.18, 134.97, 134.13, 131.42, 130.96, 129.46, 128.73, 127.26, 126.77, 126.71, 124.46, 106.77, $68.39\left(\mathrm{C}(5)\right.$ in the thiadiazole moiety), $56.16\left(\mathrm{CH}_{3} \mathrm{O}\right), 22.61$ $\left(\mathrm{CH}_{3}\right.$ in $\left.\mathrm{CH}_{3} \mathrm{CO}\right), 18.23\left(\mathrm{CH}_{3}\right)$. Found (\%): C, 58.14; $\mathrm{H}, 4.29$; $\mathrm{Cl}, 7.89 ; \mathrm{N}, 12.38 ; \mathrm{S}, 7.24 . \mathrm{C}_{22} \mathrm{H}_{19} \mathrm{ClN}_{4} \mathrm{O}_{3} \mathrm{~S}$. Calculated (\%): C, 58.08; H, 4.21; Cl, 7.79; N, 12.32; S, 7.05.

4-Acetyl-5-(2-chloro-6-methoxyquinolin-3-yl)-4,5-dihydro$\mathrm{N}$-(2,6-dimethylphenyl)-1,3,4-thiadiazole-2-carboxamide (7d). The yield was $78 \%(A), 69 \%(B)$, white powder, m.p. $153-155^{\circ} \mathrm{C}$ (EtOH). ${ }^{1} \mathrm{H}$ NMR (DMSO-d $6,300.13 \mathrm{MHz}$ ), $\delta: 10.20$ (s, $1 \mathrm{H}$, $\mathrm{NH}$ in $\mathrm{PhCONH}) ; 8.25$ (s, $1 \mathrm{H}, \mathrm{H}(4)$ in the quinoline moiety); $7.91(\mathrm{~d}, 1 \mathrm{H}, J=9.2 \mathrm{~Hz}) ; 7.59$ (d, $1 \mathrm{H}, J=2.6 \mathrm{~Hz}) ; 7.50$ (dd, $1 \mathrm{H}$, $J=9.2 \mathrm{~Hz}, J=2.7 \mathrm{~Hz}) ; 7.42(\mathrm{~s}, 1 \mathrm{H}) ; 7.14$ (s, $3 \mathrm{H}) ; 3.91$ (s, $3 \mathrm{H}$, $\left.\mathrm{CH}_{3} \mathrm{O}\right) ; 2.55\left(\mathrm{~s}, 3 \mathrm{H}, \mathrm{CH}_{3} \mathrm{CO}\right) ; 2.19\left(\mathrm{~s}, 6 \mathrm{H}, 2 \mathrm{CH}_{3}\right) .{ }^{13} \mathrm{C} \mathrm{NMR}$ (DMSO- $\left.\mathrm{d}_{6}, 75 \mathrm{MHz}\right), \delta: 170.51\left(\mathrm{C}=\mathrm{O}\right.$ in $\left.\mathrm{CH}_{3} \mathrm{CO}\right), 158.65$, $157.09,147.18,144.06,142.96,135.98,135.01,134.01,131.29$, $129.45,128.68,128.35,127.86,124.49,106.70,68.46(\mathrm{C}(5)$ in the thiadiazole moiety), $56.17\left(\mathrm{CH}_{3} \mathrm{O}\right), 22.57\left(\mathrm{CH}_{3}\right.$ in $\left.\mathrm{CH}_{3} \mathrm{CO}\right)$, $18.44\left(\mathrm{CH}_{3}\right.$ arom.), 18.23 ( $\mathrm{CH}_{3}$ arom.). Found (\%): $\mathrm{C}, 58.81$; $\mathrm{H}, 4.62 ; \mathrm{Cl}, 7.74 ; \mathrm{N}, 11.85 ; \mathrm{S}, 6.94 . \mathrm{C}_{23} \mathrm{H}_{21} \mathrm{ClN}_{4} \mathrm{O}_{3} \mathrm{~S}$. Calculated (\%): C, 58.91; H, 4.51; Cl, 7.56; N, 11.95; S, 6.84.

Synthesis of thiadiazoles $8 \mathrm{a}-\mathbf{d}$ (general procedure). A mixture of thiadiazole $\mathbf{6 a}-\mathbf{d}(1 \mathrm{mmol})$ and DDQ $(1 \mathrm{mmol})$ in ethanol $(10 \mathrm{~mL})$ was refluxed for 3-4 h (TLC monitoring, AcOEt-hexane $(1: 1))$. After cooling down to room temperature, the precipitate was filtered off and washed with aqueous ethanol $(1: 1)$. The resulting products were recrystallized from ethanol.

5-(2-Chloro-6-methoxyquinolin-3-yl)- $\mathrm{N}$-phenyl-1,3,4-thiadiazole-2-carboxamide (8a). The yield was $85 \%$, beige powder, m.p. $214-215^{\circ} \mathrm{C} .{ }^{1} \mathrm{H}$ NMR (DMSO-d 6 , $300.13 \mathrm{MHz}$ ), $\delta: 11.32$ 
(s, $1 \mathrm{H}, \mathrm{NH}$ in $\mathrm{PhCONH}) ; 9.16(\mathrm{~s}, 1 \mathrm{H}, \mathrm{H}(4)$ in the quinoline moiety); 7.98 (d, $1 \mathrm{H}, J=9.2 \mathrm{~Hz}) ; 7.88(\mathrm{~d}, 2 \mathrm{H}, J=7.9 \mathrm{~Hz})$; $7.70(\mathrm{~d}, 1 \mathrm{H}, J=2.8 \mathrm{~Hz}) ; 7.60(\mathrm{dd}, 1 \mathrm{H}, J=9.2 \mathrm{~Hz}, J=2.7 \mathrm{~Hz})$; $7.41(\mathrm{t}, 2 \mathrm{H}, J=7.9 \mathrm{~Hz}) ; 7.19(\mathrm{t}, 1 \mathrm{H}, J=7.4 \mathrm{~Hz}) ; 3.94(\mathrm{~s}, 3 \mathrm{H}$, $\left.\mathrm{CH}_{3} \mathrm{O}\right) .{ }^{13} \mathrm{C}$ NMR (DMSO-d $6,75 \mathrm{MHz}$ ), $\delta: 167.59,166.92$, 158.91, 156.55, 144.09, 143.96, 140.16, 138.10, 129.64, 129.24, $128.09,125.94,125.28,123.06,121.39,107.23,56.32\left(\mathrm{CH}_{3} \mathrm{O}\right)$. HRMS, found: $m / z$ 397.0511. Calculated for $\mathrm{C}_{19} \mathrm{H}_{14} \mathrm{ClN}_{4} \mathrm{O}_{2} \mathrm{~S}^{+}$: $397.0521[\mathrm{M}+\mathrm{H}]^{+}$.

5-(2-Chloro-6-methoxyquinolin-3-yl)- $\mathrm{N}$-(2-chlorophenyl)1,3,4-thiadiazole-2-carboxamide (8b). The yield was 93\%, gray powder, m.p. $208-209^{\circ} \mathrm{C} .{ }^{1} \mathrm{H}$ NMR (DMSO-d 6 , $300.13 \mathrm{MHz}$ ), $\delta: 10.71(\mathrm{~s}, 1 \mathrm{H}, \mathrm{NH}$ in PhCONH); $9.14(\mathrm{~s}, 1 \mathrm{H}, \mathrm{H}(4)$ in the quinoline moiety); $7.98(\mathrm{~d}, 1 \mathrm{H}, J=9.1 \mathrm{~Hz}) ; 7.84(\mathrm{~d}, 1 \mathrm{H}$, $J=8.0 \mathrm{~Hz}) ; 7.67(\mathrm{~s}, 1 \mathrm{H}) ; 7.61(\mathrm{~d}, 2 \mathrm{H}, J=8.3 \mathrm{~Hz}) ; 7.45(\mathrm{t}, 1 \mathrm{H}$, $J=7.6 \mathrm{~Hz}) ; 7.37(\mathrm{~d}, 1 \mathrm{H}, J=7.6 \mathrm{~Hz}) ; 3.96\left(\mathrm{~s}, 3 \mathrm{H}, \mathrm{CH}_{3} \mathrm{O}\right)$. ${ }^{13} \mathrm{C}$ NMR (DMSO-d $\left.6,75 \mathrm{MHz}\right), \delta: 167.19,166.66,158.91$, $156.66,144.08,143.98,140.23,134.01,130.19,129.65$, 129.03, 128.48, 128.28, 128.09, 127.64, 126.01, 123.00, 107.21, $56.33\left(\mathrm{CH}_{3} \mathrm{O}\right)$. HRMS, found: $m / z$ 431.0119. Calculated for $\mathrm{C}_{19} \mathrm{H}_{13} \mathrm{Cl}_{2} \mathrm{~N}_{4} \mathrm{O}_{2} \mathrm{~S}^{+}: 431.0131[\mathrm{M}+\mathrm{H}]^{+}$.

5-(2-Chloro-6-methoxyquinolin-3-yl)- $N$-(2-methylphenyl)1,3,4-thiadiazole-2-carboxamide (8c). The yield was $72 \%$, gray powder, m.p. $205-207^{\circ} \mathrm{C} .{ }^{1} \mathrm{H}$ NMR (DMSO-d 6 , $300.13 \mathrm{MHz}$ ), $\delta: 10.84(\mathrm{~s}, 1 \mathrm{H}, \mathrm{NH}$ in $\mathrm{PhCONH}) ; 9.14(\mathrm{~s}, 1 \mathrm{H}, \mathrm{H}(4)$ in the quinoline moiety); $7.97(\mathrm{~d}, 1 \mathrm{H}, J=9.2 \mathrm{~Hz}) ; 7.68(\mathrm{~d}, 1 \mathrm{H}$, $J=2.5 \mathrm{~Hz}) ; 7.60(\mathrm{dd}, 1 \mathrm{H}, J=9.2 \mathrm{~Hz}, J=2.7 \mathrm{~Hz}) ; 7.45(\mathrm{~d}, 1 \mathrm{H}$, $J=8.2 \mathrm{~Hz}) ; 7.37-7.20(\mathrm{~m}, 3 \mathrm{H}) ; 3.94\left(\mathrm{~s}, 3 \mathrm{H}, \mathrm{CH}_{3} \mathrm{O}\right) ; 2.31$ (s, $3 \mathrm{H}, \mathrm{CH}_{3}$ ). ${ }^{13} \mathrm{C}$ NMR (DMSO-d 6 , $75 \mathrm{MHz}$ ), $\delta: 167.35,166.88$, $158.90,156.70,144.11,143.95,140.17,135.34,134.11,130.95$, $129.65,128.10,127.21,126.88,126.66,125.96,123.09,107.18$, $56.32\left(\mathrm{CH}_{3} \mathrm{O}\right), 18.28\left(\mathrm{CH}_{3}\right)$. HRMS, found: $m / z 411.0669$. Calculated for $\mathrm{C}_{20} \mathrm{H}_{16} \mathrm{ClN}_{4} \mathrm{O}_{2} \mathrm{~S}^{+}: 411.0677[\mathrm{M}+\mathrm{H}]^{+}$.

5-(2-Chloro-6-methoxyquinolin-3-yl)- $\mathrm{N}$-(2,6-dimethylphenyl)-1,3,4-thiadiazole-2-carboxamide (8d). The yield was $70 \%$, light yellow powder, m.p. $287-288^{\circ} \mathrm{C} .{ }^{1} \mathrm{H}$ NMR (DMSO-d 6 , $300.13 \mathrm{MHz}$ ), $\delta: 10.85$ (s, $1 \mathrm{H}, \mathrm{NH}$ in $\mathrm{PhCONH}$ ); 9.15 (s, $1 \mathrm{H}$ ); $7.99(\mathrm{~d}, 1 \mathrm{H}, J=9.2 \mathrm{~Hz}) ; 7.70(\mathrm{~d}, 1 \mathrm{H}, J=2.7 \mathrm{~Hz}) ; 7.62(\mathrm{~d}, 1 \mathrm{H}$, $J=9.2 \mathrm{~Hz}) ; 7.19-7.13(\mathrm{~m}, 3 \mathrm{H}) ; 3.95(\mathrm{~s}, 3 \mathrm{H}) ; 2.24(\mathrm{~s}, 6 \mathrm{H}$, $\left.2 \mathrm{CH}_{3}\right) .{ }^{13} \mathrm{C}$ NMR (DMSO-d $\left.6,75 \mathrm{MHz}\right), \delta: 167.19,166.89$, $158.93,156.58,144.18,143.97,140.28,136.01,134.33,129.69$, $128.34,128.14,127.78,125.98,123.19,107.21,56.35\left(\mathrm{CH}_{3} \mathrm{O}\right)$, $18.53\left(\mathrm{CH}_{3}\right)$. HRMS, found: $\mathrm{m} / z$ 425.0834. Calculated for $\mathrm{C}_{21} \mathrm{H}_{18} \mathrm{ClN}_{4} \mathrm{O}_{2} \mathrm{~S}^{+}: 425.0834[\mathrm{M}+\mathrm{H}]^{+}$.
This paper does not contain descriptions of studies on animals or humans.

The authors declare no competing interests.

\section{References}

1. L. M. Nainwal, S. Tasneem, W. Akhtar, G. Verma, M. F. Khan, S. Parvez, M. Shaquiquzzaman, M. Akhter, M. M. Alam, Eur. J. Med. Chem., 2019, 164, 121.

2. S. Kumar, S. Bawa, H. Gupta, Mini-Rev. Med. Chem., 2009, 9, 1648.

3. Order of the Government of the Russian Federation, April 16, 2020, Order. No. 1030-r.

4. W. S. Hamama, M. E. Ibrahim, A. A. Gooda, H. H. Zoorob, RSC Adv., 2018, 8, 8484.

5. B. F. Abdel-Wahab, R. E. Khidre, J. Chem., 2013, ID 851297; DOI: $10.1155 / 2013 / 851297$.

6. Y. Hu, C.-Y. Li, X.-M. Wang, Y.-H. Yang, H.-L. Zhu, Chem. Rev., 2014, 114, 5572.

7. L. Joseph, M. George, P. Mathews, J. Pharm. Chem. Biol. Sci., 2015, 3, 329.

8. A. R. Bhat, Tazeem, A. Azam, I. Choi, F. Athar, Eur. J. Med. Chem., 2011, 46, 3158.

9. S. B. Marganakop, R. R. Kamble, T. Taj, M. Y. Kariduraganvar, Med. Chem. Res., 2012, 21, 185.

10. S. B. Marganakop, R. R. Kamble, J. Hoskeri, D. J. Prasad, G. Y. Meti, Med. Chem. Res., 2014, 23, 2727.

11. M. M. Krayushkin, V. N. Yarovenko, I. V. Zavarzin, Russ. Chem. Bull., 2019, 68, 1143.

12. M. M. Krayushkin, V. N. Yarovenko, I. V. Zavarzin, Russ. Chem. Bull., 2004, 53, 517.

13. V. N. Yarovenko, A. V. Shirokov, O. N. Krupinova, I. V. Zavarzin, A. V. Ignatenko, M. M. Krayushkin, Chem. Heterocycl. Compd. (Engl. Transl.), 2003, 39, 1633.

14. K. A. Myannik, V. N. Yarovenko, G. M. Rodionova, T. K. Baryshnikova, M. M. Krayushkin, Arkivoc, 2017, iii, 316.

Received December 22, 2020; in revised form February 4, 2021; accepted March 29, 2021 